\title{
Desarrollo de competencias y habilidades a través de proyectos basados en empresas reales: Análisis en asignaturas de Dirección de Operaciones
}

\author{
Teaching Operations Management through real case studies: \\ development of skills and competences
}

José Antonio Alfaro Tanco (jalfaro@unav.es)

Victoria Rodríguez Chacón (vrodriguez@unav.es)

Universidad de Navarra (España)

\author{
Itxaso Amorrortu Gervasio (iamorrortu@eps.mondragon.edu) \\ Universidad de Mondragón (España) \\ http://dx.doi.org/10.12795/EDUCADE.2014.105.03
}

\begin{abstract}
RESUMEN: El trabajo que aquí se presenta tiene como objetivo lograr que el alumno adquiera los conocimientos básicos de la Dirección de Operaciones, a la vez que desarrolle competencias necesarias para su incorporación en el mercado laboral y establezca un contacto real con la empresa. La base metodológica de la propuesta que aquí se presenta es la formación por proyectos como una metodología efectiva para lograr una educación basada en competencias, y que integra la adquisición de conocimientos con el desarrollo de habilidades y valores.
\end{abstract}

Los modelos de aprendizaje basados en proyectos tienen su base científica en el proceso de generación de conocimiento, en el cual los estudiantes no son receptores pasivos de conocimiento, sino que construyen conocimiento sobre la base de lo que ellos realmente saben y han experimentado, lo que se consigue a través de la participación e interacción con otros. Los autores de este artículo muestran los resultados obtenidos en los últimos seis años a través de la experiencia en asignaturas de Operaciones.

Tal y como se observa en los resultados, mediante la utilización de la metodología descrita el aprendizaje de los contenidos se resuelve de una manera exitosa. El plantearle al alumno que resuelva un problema en una empresa real de una manera semi-autónoma hace que éste se plantee cuestiones que con las clases magistrales no lo haría. Además, debido al seguimiento realizado a través de los hitos por el profesor, los equipos son capaces de corregir sus errores y el conocimiento de la materia se refuerza.

PALABRAS CLAVE: Dirección de Operaciones, Casos reales, Aprendizaje basado en proyectos, Competencias, Habilidades.

ABSTRACT: The main aim of our work is to show how a student may get competences and skills through the development of real case studies. We implement this study in Operations Management courses of different Spanish universities. The methodology we purpose is based on learning projects, which permits that students actively generate knowledge through their experiences in real firms.

Experiencia docente. Recibido: 30-05-13 - Versión revisada: 15-07-13, Aceptado: 05-09-13, Publicado on-line 03-01-14 Licencia Creative Commons BY NC ND · 2014 · Asociación Española de Contabilidad y Administración de Empresas - AECA 
As we show in our empirical analysis, the results are relevant: the fact that students have to solve a problem in a real firm makes them face questions that are difficult they challenge in theoretical sessions. Besides, the control of the professor is key so that the teams may rectify and the understanding of the course be reinforced.

KEYWORDS: Operations Management, Case studies, Learning based on projects, Skills, Abilities.

\section{INTRODUCCIÓN}

Son muchas las asociaciones y organismos internacionales que demandan cambios en el sistema educativo, entre ellas la Organización Internacional del Trabajo, la cual recomienda que "se asegure el desarrollo y consolidación de sistemas de educación y formación profesional que ofrezcan oportunidades adecuadas para el desarrollo y la certificación de las competencias que requiere el mercado de trabajo" (Pronko, 2005). Además, organizaciones como la Comisión Mundial sobre la Dimensión Social de la Globalización (CMDSG) considera la educación como "un elemento esencial dentro de una economía global en la que la formación, la calificación y el conocimiento tienen cada vez más importancia, ya no para el éxito, sino para la supervivencia económica" (CMDSG, 2004).

En respuesta a estos requerimientos, organismos como la UNESCO, La Unión Europea, el Instituto Internacional de la UNESCO para la Educación Superior en América Latina y el Caribe (IESALC) o la Organización de Estados Iberoamericanos (OEI) han desarrollado una serie de iniciativas en las que se persiguen principalmente dos objetivos: reducir la brecha entre la teoría y la realidad, y desarrollar mecanismos de convergencia de los sistemas de educación.

En Europa dentro de la iniciativa de la construcción del Espacio Europeo de Educación Superior (EEES) que se inició en 1998 y en la que actualmente participan un total de 45 países, se han desarrollado diferentes proyectos, dentro de los cuales, cabe destacar el proyecto Tunning Educational Structures in Europe. Este proyecto, dentro del Área Temática de Administración y Dirección de Empresas, propone facilitar al estudiante la adquisición de habilidades transferibles mediante actividades como proyectos, tesinas y juegos de empresa. El objetivo es desarrollar aquellas competencias necesarias para cerrar el espacio entre la teoría y la realidad, competencias que han sido siempre demandadas pero que aún representan un problema para muchos graduados al enfrentarse a su entrada al mercado de trabajo.

Se han propuesto muchas alternativas docentes para desarrollar las competencias necesarias en los alumnos de Administración y Dirección de Empresas, dentro de las cuales una de las más difundidas es el método del caso (Jackson, 2004). En esencia, en la metodología del caso se describe una situación actual, que normalmente requiere la toma de decisiones, un reto, una oportunidad y un problema que enfrenta una persona (o personas) en una organización (Mauffette-Leenders et al., 1997, p. 2). Con el uso de esta metodología se pretende desarrollar en los alumnos competencias interpersonales, de análisis, habilidades para la toma de decisiones y relacionar la teoría con la práctica. En la discusión de un caso, el profesor normalmente es un facilitador que anima a los alumnos a definir el problema, sugerir diferentes alternativas de solución, hacer recomendaciones y aplicar las conclusiones a otras empresas o contextos. (Barnes et al., 1994a, 1994b; Christensen, 1991; Erskine et al., 1998; Welty, 1989). 
Lo más frecuente es que los casos se entreguen a los alumnos impresos en papel, aunque en la actualidad muchos de ellos se presentan con medios audiovisuales. En el caso se describe con mucho detalle la empresa y su situación, lo que permite un acercamiento valioso del alumno al medio empresarial, pero no permite un acercamiento físico del alumno a la empresa.

La actividad que aquí se presenta, la formación por proyectos, tiene como objetivo lograr que el alumno adquiera los conocimientos básicos de la Gestión de Operaciones, a la vez que desarrolla competencias necesarias para su incorporación en el mercado laboral y establece un contacto real con la empresa. La misma ha sido propuesta por muchos autores (Chinnowsky et al., 2006; Gijselaers, 1996; Johnson, 1999; Padmanadhan and Katti, 2002) como una metodología efectiva para lograr una educación basada en competencias (Parsons et al., 2005; Mulcahy, 2000; Kelly, 2007) y que integra la adquisición de conocimientos con el desarrollo de habilidades y valores. Los modelos de aprendizaje basados en proyectos tienen su base científica en el proceso de generación de conocimiento, en el cual los estudiantes no son receptores pasivos de conocimiento (Chinnowsky et al., 2006), sino que construyen conocimiento sobre la base de lo que ellos realmente saben y han experimentado, lo que se consigue a través de la participación e interacción con otros (Gijselaers, 1996). Existen estudios donde se muestran cómo los estudiantes mejoran el grado de adquisición de conocimientos a través de proyectos basados en el aprendizaje (Jackson et al., 2012).

Los autores de este artículo han trabajado los últimos años en facultades de Económicas y Empresariales y de Ingeniería aplicando la metodología y la orientación docente que aquí se propone, siempre en asignaturas relacionadas con la gestión. En total los autores han aplicado la metodología en 12 grupos durante los últimos 6 años.

La actividad que se propone se presenta de manera general en el apartado 2. En el siguiente, se explica la originalidad y contraste con las iniciativas normalmente desarrolladas, enfatizando el esfuerzo que supone para el profesor poner en marcha esta iniciativa, y los problemas que ha habido que ir resolviendo a medida que el curso avanza. En el apartado 4 se analizan, por un lado, las competencias sobre las que incide esta actividad y cómo esta actividad incide en la capacidad de desarrollo de estrategias empresariales, y, por otro lado, los resultados obtenidos en los dos últimos cursos. Finalmente, se exponen las conclusiones generales de la puesta en marcha de esta iniciativa, algunas de las limitaciones del trabajo, y algunas líneas futuras de investigación.

\section{DESCRIPCIÓN GENERAL DE LA INICIATIVA}

El objetivo de la asignatura es lograr que los alumnos conozcan y sean capaces de proponer mejoras en la Gestión de las Operaciones de una empresa. Para ello se da una base teórica sobre la Gestión de Operaciones que luego los alumnos enriquecerán a través del desarrollo de un proyecto. Las operaciones que se estudian dentro de la asignatura son el diseño y desarrollo de un nuevo producto, el mantenimiento, la gestión de la producción y la logística de aprovisionamiento y distribución.

La asignatura se imparte a lo largo de 15 semanas, cada semana tiene asignadas 3 horas de clase. Las horas de la asignatura se dividen en horas lectivas, horas de tutoría y horas de trabajo. El proyecto se lanza la primera semana de clase, durante las 3 primeras semanas se dan tres horas de teoría en las que se da a los alumnos una visión general de las operaciones con las que tendrán que trabajar a lo largo del proyecto. En las siguientes 9 semanas los alumnos trabajan exclusivamente en el proyecto y entregan dos informes de avance; en estas semanas el único contacto entre los alumnos y el profesor son las sesiones de tutoría. En la semanas 12 y 13 los alumnos 
entregan el informe final y hacen las presentaciones de sus trabajos ante los demás grupos. En las semanas 14 y 15 se realizan dos sesiones de cierre en las que se retoma el tema introducido en las 3 primeras semanas, pero esta vez se utiliza el trabajo realizado por los alumnos como material de análisis y discusión.

El objetivo del proyecto es que los alumnos conozcan cómo se gestionan realmente las operaciones en una empresa, analicen dicha gestión con sentido crítico de manera que sean capaces de proponer mejoras en su gestión y sean capaces de valorar las mejoras que proponen.

En la presentación del proyecto se establecen 3 hitos. A continuación se describen brevemente las actividades y objetivos docentes de cada hito.

i.Hito 1: El objetivo de este hito es definir la actividad de la empresa en la que se centrará el proyecto. Las actividades que llevan a cabo los alumnos son: definir la empresa, buscar información sobre la misma, buscar información en libros, revistas especializadas e Internet sobre las operaciones que se van a analizar, definir y planificar en el desarrollo del proyecto y planificar la entrevista a la empresa mediante el desarrollo de un guion, con base en la información obtenida. Al finalizar este hito los alumnos deben entregar un informe técnico de 5 páginas que debe contener un resumen de la información encontrada y el guion de la entrevista.

ii.Hito 2: El objetivo de este hito es proponer mejoras sobre las distintas operaciones de la empresa. En este hito los alumnos deben visitar la empresa elegida y como resultado de la visita redactar un informe técnico en el que se describan las operaciones de la empresa y los posibles puntos a mejorar. Al finalizar este hito los alumnos deben presentar un informe de 5 páginas.

iii.Hito 3: El objetivo de este último hito es valorar las distintas propuestas desde el punto de vista económico y operativo. En este hito los alumnos deben valorar las necesidades económicas para poner en marcha esta mejora y sus beneficios, además deben prever los problemas que conllevaría su implantación. Al finalizar este hito los alumnos presentan el informe final del proyecto, en el que deben incluir la descripción de las operaciones de la empresa, los puntos de mejora identificados, las propuestas de mejora y su valoración. Dicho informe no debe tener una extensión de más de 15 páginas.

Después de la entrega de cada hito los alumnos presentan en el aula los resultados obtenidos hasta el momento, por lo tanto además de desarrollar la expresión escrita en las memorias, la expresión oral es puesta en práctica.

El proyecto lo desarrollan los alumnos en grupos de 4 o 5 estudiantes. En cada hito, los grupos tienen una sesión individual de tutoría con el profesor y reciben un informe por parte del profesor con los comentarios sobre el informe técnico entregado.

En la primera sesión de tutoría el profesor analiza con los estudiantes la planificación del proyecto y la orientación del mismo, mientras que en la segunda y tercera sesión se analizan los resultados de los informes anteriores, se revisa la orientación del proyecto y se planifican las tareas a realizar.

Se propone un calendario para el desarrollo de la asignatura a lo largo del cuatrimestre, planificación que permite controlar el esfuerzo por parte del profesor de manera que pueda utilizar la metodología eficaz y eficientemente. Una planificación tipo se puede ver en la Tabla 1. 
José Antonio Alfaro, Victoria Rodríguez, Itxaso Amorrortu

Desarrollo de competencias de habilidades a través de proyectos en empresas reales

TABla 1 Planificación del desarRollo de la iniciativa

\begin{tabular}{|c|c|}
\hline Semana & Actividad \\
\hline 1 & Lanzamiento y clase \\
\hline 2 & Clase \\
\hline 3 & Clase - Reuniones de seguimiento \\
\hline 4 & Entrega del hito 1 \\
\hline 5 & Reuniones de seguimiento \\
\hline 6 & Clase \\
\hline 7 & Clase \\
\hline 8 & Entrega del hito 2 \\
\hline 9 & Reunión de seguimiento \\
\hline 10 & Clase \\
\hline 11 & Clase \\
\hline 12 & Entrega del hito 3 \\
\hline 13 & Presentaciones finales \\
\hline 14 & Clase \\
\hline 15 & Clase \\
\hline
\end{tabular}

Fuente: Elaboración propia

\section{ORIGINALIDAD Y CONTRASTE CON LAS INICIATIVAS NORMALMENTE DESARROLLADAS}

La originalidad de esta propuesta está en el enfoque que los alumnos deben dar al proyecto, la forma de evaluar y hacer el seguimiento. Desde el punto de vista de la orientación, al pedir a los alumnos que propongan mejoras en alguna de las operaciones se les está obligando a hacer un análisis con sentido crítico. Respecto a la forma de evaluación, se valora el alcance de varias competencias, tal que la forma de evaluar que se propone permite identificar al estudiante que no haya trabajado si se da el caso a través de las reuniones de seguimiento. En cuanto al seguimiento, las tutorías de seguimiento son obligatorias y deben asistir todos los miembros del grupo. En estas reuniones se puede detectar el nivel de implicación de los alumnos con preguntas específicas sobre cada tema. Además los grupos reciben dos informes de evaluación intermedios.

El objetivo de estos informes es que los grupos puedan hacer correcciones sobre los informes de avance y entregar un buen informe final del proyecto. 


\subsection{CARGA De tRABAJO}

La actividad que aquí se propone no requiere ningún gasto o inversión adicional para la universidad más que la formación de los profesores que la vayan a utilizar. En lo que a la dedicación horaria del profesor respecta, a modo de ejemplo, la siguiente tabla recoge los cálculos de ésta en el caso de tener 15 grupos de 5 alumnos (75 alumnos en total).

TABLA 2. CARGA HORARIA APROXIMADA DEL PROFESOR

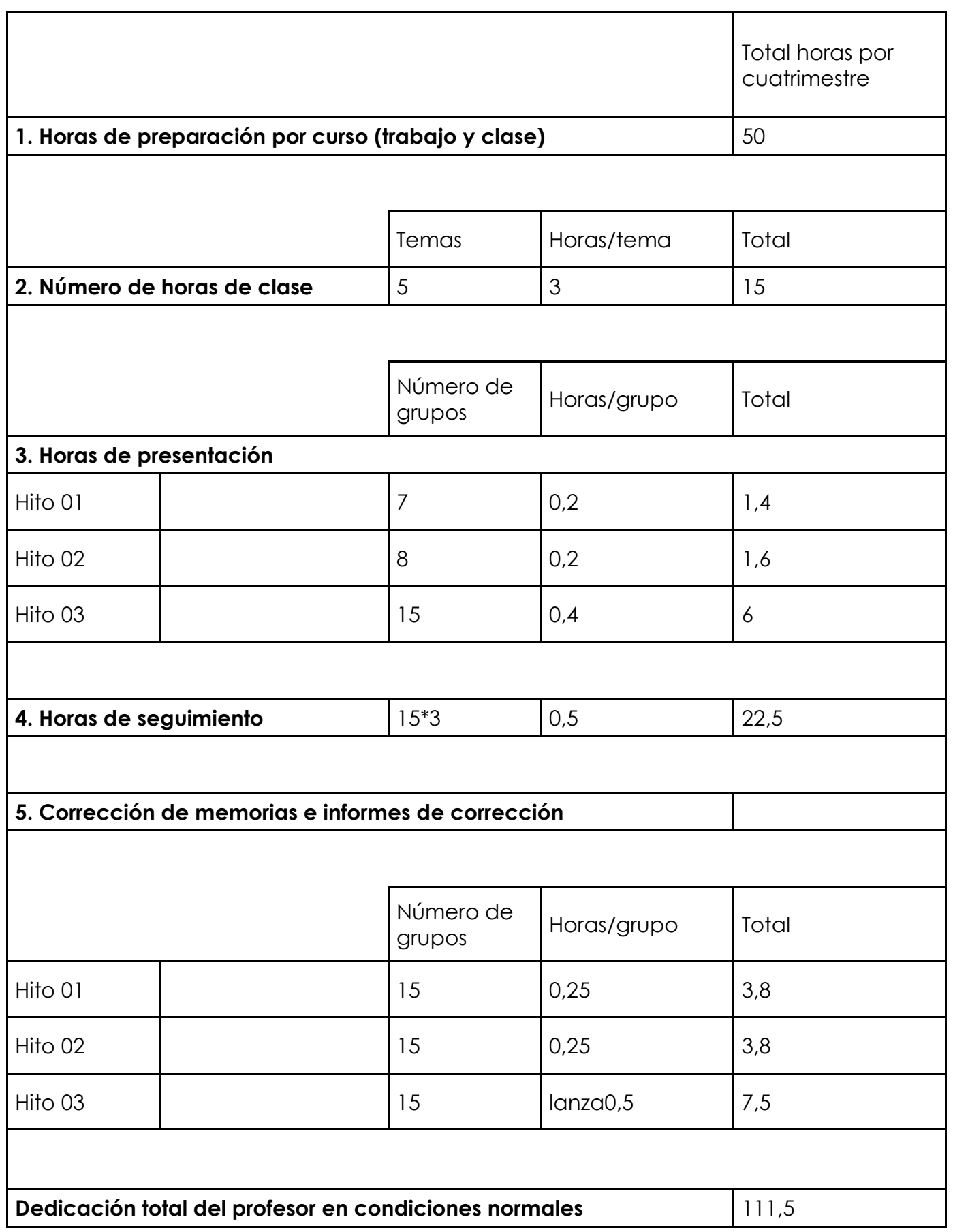

Fuente: Elaboración propia 
A esta dedicación habría que sumarle el tiempo de preparación y corrección de exámenes y el tiempo que supondría para el profesor contactar con alguna empresa cuando se presentan grupos especiales que no tienen posibilidad de contactar con ninguna empresa. En este caso, hay que destacar que cada vez es mayor el número de alumnos de intercambio, los cuales suelen tener problemas para contactar con empresas locales dispuestas a colaborar en el trabajo. En este tipo de situaciones, el profesor contacta directamente con varias empresas para explicarles el objetivo del trabajo, y conocer su actitud de colaboración. También se les explica que serán alumnos de intercambio los que realizarán el trabajo, con objeto de que les ayuden con el idioma y podamos asegurarnos de que los estudiantes puedan asimilar los conocimientos dados por la empresa. Además, en esta situación se intenta encontrar empresas con perfil internacional, lo cual hace que el tiempo invertido en este tipo de situaciones sea tan elevado.

\subsection{Dificultades PARA LA PUESTA EN MARCHA DE LA INICIATIVA}

Son varias las dificultades que se han identificado en estos años de puesta en práctica de la metodología de proyectos. De forma más específica, estas son los problemas burocráticos para conseguir que todos los alumnos puedan comenzar de forma simultánea; la gestión de las reuniones de seguimiento; compaginar resultados y expectativas de las empresas; y problemas no detectados dentro del grupo. Seguidamente, desarrollamos cada uno de ellos.

De acuerdo con la planificación de la asignatura el proyecto debería lanzarse el primer día de clase y en una situación ideal los grupos deberían quedar constituidos la segunda semana de clase. Sin embargo se dan varios casos en los que los alumnos formalizan la matricula durante la segunda o tercera semana de clase. Este hecho supone una dificultad de gestión, sobre todo, cuando se concentran muchos alumnos de intercambio en esta situación, pues es difícil integrarlos en los grupos ya que la mayoría ya están completos y si se forma un grupo sólo con estos alumnos es difícil establecer y mantener el contacto con la empresa. Lo que se intenta es integrar a estos alumnos dentro de los grupos ya establecidos. La experiencia cuando todo el grupo lo forman estudiantes de intercambio no suele ser positiva.

En un principio las reuniones de seguimiento supusieron un verdadero trastorno para el trabajo regular del profesor, pues los alumnos concertaban una cita con el profesor a cualquier hora de la semana establecida para hacer las reuniones de seguimiento y esto hacía que las reuniones se dispersaran a lo largo de toda la semana. Para solucionar este inconveniente se propone a los alumnos dos o tres bloques horarios en los que los grupos se deben apuntar para hacer la reunión de seguimiento. Esta nueva forma de trabajar permite al profesor concentrar la dedicación a las reuniones de seguimiento con el incremento de eficiencia que ello supone.

Otra dificultad que los autores encontraron en experiencias anteriores fue la de compaginar los objetivos académicos con las expectativas que las empresas se creaban con el proyecto. Este problema se resuelve aclarando tanto a las empresas como a los alumnos que los objetivos académicos priman sobre los objetivos de la empresa, pues a través de este trabajo se busca que los alumnos aprendan una metodología de trabajo y no que hagan un trabajo de consultoría.

Por último, puede ocurrir que el profesor no alcance a detectar problemas que el grupo no es capaz de resolver y convive con ellos hasta finalizar el proyecto. Este hecho puede suponer un impedimento para alcanzar proyectos de alta calidad, pero por otra parte permite que los alumnos trabajen en el desarrollo de su capacidad para gestionar conflictos. En situaciones de este tipo, el grupo debe asumir sus bajos 
resultados e intentar mejorar la nota en el resto de pruebas: no hay opción a alargar el trabajo en este tipo de situaciones.

\section{BENEFICIOS PARA EL ALUMNO EN LA METODOLOGÍA DE PROYECTOS DE EMPRESA}

Dos son los indicadores que hemos utilizado para determinar la utilidad de proyectos basados en empresas reales: por un lado, el desarrollo de competencias por parte de los alumnos, y, por otro lado, la adquisición de conocimientos teóricos, medida a través de los resultados finales.

\subsection{DeSARROLlo de COMPETENCIAS DENTRO de LA ELABORACIÓN DEL PROYECTO}

Esta sección es clave dentro del artículo, en cuanto que permite justificar la utilización de esta metodología. A través de la iniciativa que aquí se describe se pretende contribuir en el desarrollo de 14 competencias genéricas de las 27 identificadas en el proyecto Tunning Educational Structures in Europe, competencias que se han ido desarrollando a lo largo de la carrera.

En la Tabla 3 se puede ver la relación entre las actividades que comprenden la actividad y la incidencia en dichas competencias.

TABLA 3. COMPETENCIAS SOBRE LAS QUE INCIDE LA INICIATIVA

\begin{tabular}{|c|c|c|c|c|c|c|c|c|c|c|c|c|c|c|c|}
\hline & & 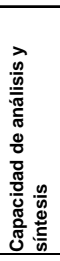 & 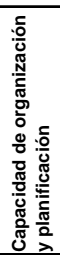 & 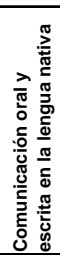 & 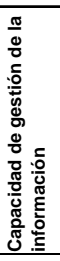 & 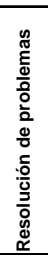 & 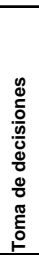 & 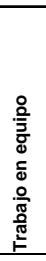 & 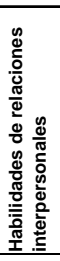 & 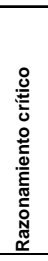 & 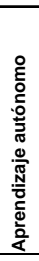 & 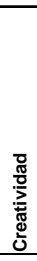 & 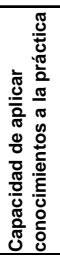 & 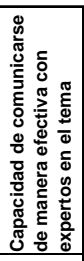 & 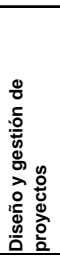 \\
\hline \multirow{4}{*}{ 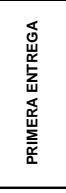 } & Planificación del proyecto & $\mathrm{x}$ & $\mathrm{x}$ & & & & & $\mathbf{x}$ & & & & & & & $x$ \\
\hline & \begin{tabular}{|l|l|}
$\begin{array}{l}\text { Busqueda orientada de teoria } \\
\text { sobre las operaciones }\end{array}$ & \\
\end{tabular} & & & & $\mathrm{x}$ & & & & & & $\mathrm{x}$ & & & & \\
\hline & $\begin{array}{l}\text { Búsqueda de información sobre la } \\
\text { actividad de la empresa }\end{array}$ & & & & $\mathrm{x}$ & & & & & & $\mathrm{x}$ & & & & \\
\hline & \begin{tabular}{|l}
$\begin{array}{l}\text { lanificación de la visita a la } \\
\text { empresa }\end{array}$ \\
\end{tabular} & $\mathrm{x}$ & $\mathrm{x}$ & & & & $\mathrm{x}$ & & & $\mathrm{x}$ & $\mathrm{x}$ & & $\mathrm{x}$ & & \\
\hline \multirow{3}{*}{ 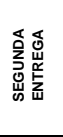 } & Visita a la empresa & & & & & & & & $\mathbf{x}$ & & & & & $\mathbf{x}$ & \\
\hline & \begin{tabular}{|l}
$\begin{array}{l}\text { Identificación de puntos de } \\
\text { mejora }\end{array}$ \\
\end{tabular} & $\mathrm{x}$ & & & & & $\mathrm{x}$ & & & $\mathrm{x}$ & & & $\mathrm{x}$ & & \\
\hline & $\begin{array}{l}\begin{array}{l}\text { Planteamiento de propuestas de } \\
\text { mejora }\end{array} \\
\end{array}$ & $\mathrm{x}$ & & & & $\mathrm{x}$ & $\mathrm{x}$ & & & & & $\mathrm{x}$ & $\mathrm{x}$ & $\mathrm{x}$ & \\
\hline \multirow{3}{*}{ 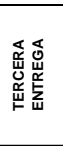 } & $\begin{array}{l}\text { Profundización en las propuestas } \\
\text { de mejora }\end{array}$ & $\mathrm{x}$ & & & & $\mathrm{x}$ & $\mathbf{x}$ & & & & & $\mathrm{x}$ & $\mathrm{x}$ & $\mathrm{x}$ & \\
\hline & $\begin{array}{l}\text { Valoración de las propuestas de } \\
\text { mejora }\end{array}$ & & & & & & $\mathrm{x}$ & & & $\mathrm{x}$ & & & & & \\
\hline & $\begin{array}{l}\text { Planteamiento de posibles } \\
\text { problemas asociados a la }\end{array}$ & $\mathrm{x}$ & & & & & & & & $\mathrm{x}$ & & & & & \\
\hline \multirow{2}{*}{ 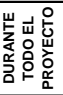 } & $\begin{array}{l}\text { Entrega de informes de avance e } \\
\text { informe final }\end{array}$ & & & $\mathbf{x}$ & & & & & & & & & & $\mathrm{x}$ & \\
\hline & \begin{tabular}{|lll}
$\begin{array}{l}\text { Presentación de informes en } \\
\text { público }\end{array}$ & en \\
\end{tabular} & & & $\mathrm{x}$ & & & & & & & & & & $\mathrm{x}$ & \\
\hline
\end{tabular}

Fuente: Elaboración propia

En la primera entrega, son la capacidad de análisis, la capacidad de organización y planificación, la capacidad de gestión de la información, el trabajo en equipo y el aprendizaje autónomo las capacidades que se consiguen desarrollar. En esta fase, la planificación del proyecto y la planificación de la visita a la empresa son claves para el desarrollo de estas competencias.

Respecto a la segunda entrega, la visita a la empresa y el planteamiento de propuestas de mejoras son las actividades que permiten a los alumnos desarrollar capacidades, especialmente, la capacidad de análisis y síntesis, la resolución de problemas, la toma de decisiones y la capacidad de aplicar conocimientos a la práctica. 
En cuanto a la tercera entrega, es la tarea de profundización de propuestas de mejora la que ayuda a conseguir un mayor número de competencias, tales como la tomas de decisiones, la creatividad y la resolución de problemas.

Resumiendo, cabe destacar la incidencia que tienen la preparación y visita a la empresa, la identificación de los puntos de mejora, las propuestas de mejora y la valoración de las mismas en el desarrollo de competencias que ayudarán al alumno a enfrentarse al mercado laboral.

La visita a la empresa exige una gran preparación de la misma, ya que por una parte, antes de hacer la visita los alumnos deben conocer la actividad de la empresa, buscar información y hacerse una idea de cómo podría ser el proceso de diseño y desarrollo de nuevos productos, el proceso productivo, las actividades logísticas y las actividades relacionadas con la gestión del mantenimiento. Por otra parte, la visita a la empresa es en muchos casos el primer acercamiento profesional de los alumnos a una empresa. En dicha visita los alumnos deben estar en capacidad de contrastar la información de la que disponen y además obtener la mayor cantidad de conocimientos relacionados con su proyecto, por lo tanto, deben ser ellos quienes guíen el desarrollo de la reunión.

La identificación de los puntos de mejora contribuye sobre todo al desarrollo de la capacidad crítica del alumno. Este es el apartado con mayor dificultad a la que se enfrentan los alumnos, pues con una sola visita deben conocer una empresa y además identificar puntos débiles. Para hacer frente a este apartado se recomienda a los alumnos que hagan una búsqueda previa en Internet con frases como "problemas logísticos en el sector de la construcción", de esta forma cuando acuden a la visita van con ideas sobre posibles problemas y pueden trabajar estos temas con la persona que les está atendiendo.

El desarrollo de propuestas de mejora y su valoración pone a prueba la capacidad creativa de los alumnos ya que, por una parte, deben plantear formas alternativas de gestionar y por la otra deben valorar económicamente dichas propuestas. Además dichas propuestas se deben valorar desde el punto de vista de la gestión, por lo tanto, los estudiantes deben prever los posibles problemas que conllevaría la implantación de dicha mejora.

Consideramos que las metodologías tradicionales de realización de trabajos en empresas se basan en la lectura de artículos o en la descripción de una visita concreta. El seguimiento continuo, la interacción repetida con los alumnos y el análisis crítico son claves para conseguir desarrollar las capacidades descritas en la Tabla 3, y que, a su vez, justifican el desarrollo de la metodología de proyectos.

\subsection{RESULTADOS EN NOTAS OBTENIDAS POR LOS ALUMNOS}

En los cursos 09-10, 10-11 y 11-12 la asignatura Dirección de Operaciones se ha impartido con la metodología que aquí se propone, los resultados obtenidos se han comparado con los resultados obtenidos en los tres cursos anteriores. En la Tabla 4 se puede ver el número de alumnos por curso.

TABLA 4. NÚMERO DE ALUMNOS POR CURSO

\begin{tabular}{|c|c|c|c|c|c|c|}
\hline & $\begin{array}{c}\text { Curso } \\
\mathbf{0 6 - 0 7}\end{array}$ & $\begin{array}{c}\text { Curso } \\
\mathbf{0 7 - 0 8}\end{array}$ & $\begin{array}{c}\text { Curso } \\
\mathbf{0 8 - 0 9}\end{array}$ & $\begin{array}{c}\text { Curso } \\
\mathbf{0 9 - 1 0}\end{array}$ & $\begin{array}{c}\text { Curso } \\
\mathbf{1 0 - 1 1}\end{array}$ & $\begin{array}{c}\text { Curso } \\
\mathbf{1 1 - 1 2}\end{array}$ \\
\hline $\begin{array}{c}\text { Número de } \\
\text { alumnos }\end{array}$ & 103 & 112 & 118 & 116 & 60 & 60 \\
\hline
\end{tabular}

Fuente: Elaboración propia. 
En la primera fase del proyecto la implicación de los alumnos en el proyecto no es mucha. De hecho las notas correspondientes al primer hito suelen ser muy bajas, pero a partir del segundo hito los alumnos demuestran mayor interés por el proyecto, acuden a las reuniones de seguimiento con material más elaborado y consiguen alcanzar notas relativamente altas tal y como se puede ver en la Tabla 5. Se puede decir que la calidad de los trabajos finales es muy buena. El hecho del incremento de la implicación de los alumnos en el proyecto podría tener dos explicaciones, la primera de ellas es que en un principio a los estudiantes les cuesta comprender y adoptar la metodología de trabajo y la segunda es que al ver notas tan bajas caen en la cuenta de la importancia de trabajar en el proyecto.

TABLA 5. RESUltAdOS OBTENIDOS EN LOS DIFERENTES HITOS

\begin{tabular}{|l|c|c|c|c|}
\hline \multicolumn{5}{|c|}{ Cursos 09-10, 10-11 y 11-12 } \\
\hline & Hito 1 & Hito 2 & Hito 3 & $\begin{array}{c}\text { Nota final } \\
\text { del trabajo }\end{array}$ \\
\hline Nota media & 4,14 & 7,26 & 8,01 & 8,28 \\
\hline $\begin{array}{l}\text { Desviación } \\
\text { estándar }\end{array}$ & 1,44 & 1,72 & 1,39 & 0,98 \\
\hline
\end{tabular}

Fuente: Elaboración propia

En lo relativo a las notas medias obtenidas (ver Tabla 6), se puede ver que las notas medias finales de la asignatura han mejorado ostensiblemente con la aplicación de la metodología descrita pero también se observa que la dispersión de los resultados es menor.

TABLA 6. RESULTADOS FINALES OBTENIDOS POR LOS ALUMNOS

\begin{tabular}{|c|c|c|c|c|c|c|}
\hline & $\begin{array}{c}\text { Curso } \\
\mathbf{0 6 - 0 7}\end{array}$ & $\begin{array}{c}\text { Curso } \\
\mathbf{0 7 - 0 8}\end{array}$ & $\begin{array}{c}\text { Curso } \\
\mathbf{0 8 - 0 9}\end{array}$ & $\begin{array}{c}\text { Curso } \\
\mathbf{0 9 - 1 0}\end{array}$ & $\begin{array}{c}\text { Curso } \\
\mathbf{1 0 - 1 1}\end{array}$ & $\begin{array}{c}\text { Curso } \\
\mathbf{1 1 - 1 2}\end{array}$ \\
\hline Nota media & 6,2 & 6,16 & 5,12 & 7,42 & 7,07 & 6,33 \\
\hline $\begin{array}{c}\text { Desviación } \\
\text { estándar }\end{array}$ & 1,72 & 1,43 & 2,2 & 1,26 & 0,4 & 1,43 \\
\hline
\end{tabular}

Fuente: Elaboración propia

Este resultado apoya la opinión de muchos profesores que afirman que al utilizar este tipo de metodologías los resultados finales de los estudiantes se concentran más en un rango relativamente alto de notas, pero a su vez, hace más difícil que los alumnos realmente capaces destaquen sobre el grupo y permite a los alumnos poco trabajadores pasar desapercibidos más fácilmente. De todas formas, la impresión de los autores es que cuando se aplican este tipo de metodologías se consigue "enganchar" en la asignatura a un mayor número de estudiantes que trabajan de forma constante.

En el curso 10-11 el examen final contenía cuatro tipos de preguntas: preguntas de teoría, una pregunta de análisis de un caso genérico, preguntas de teoría aplicada a todas las empresas estudiadas por los alumnos y preguntas específicas sobre la empresa que cada grupo trabajó; la nota de este último apartado es parte de la nota del trabajo. En la

Tabla 7 se presenta un resumen de los resultados obtenidos, en la primera fila se presentan los pesos que tienen los diferentes apartados en la nota final, en la fila Nivel alcanzado aparece el porcentaje de superación de cada apartado y en la fila Desviación estándar se presenta la dispersión de dichos resultados. 
TABLA 7. Distribución de los Resultados

\begin{tabular}{|c|c|c|c|c|c|}
\cline { 2 - 6 } & \multicolumn{2}{c|}{ Nota Examen Final } & & \multicolumn{2}{c|}{ Nota del trabajo } \\
\cline { 2 - 6 } & Teoría & $\begin{array}{c}\text { Teoría } \\
\text { aplicada a } \\
\text { la empresa }\end{array}$ & $\begin{array}{c}\text { Solución del } \\
\text { caso } \\
\text { propuesto }\end{array}$ & $\begin{array}{c}\text { Preguntas } \\
\text { sobre su } \\
\text { trabajo }\end{array}$ & $\begin{array}{c}\text { Informe final y } \\
\text { desarrollo del } \\
\text { proyecto }\end{array}$ \\
\hline $\begin{array}{c}\text { Porcentaje que } \\
\text { supone en la nota } \\
\text { definitiva }\end{array}$ & $13,00 \%$ & $13,00 \%$ & $24,00 \%$ & $10,00 \%$ & $40,00 \%$ \\
\hline Nivel alcanzado & $15,60 \%$ & $11,60 \%$ & $60 \%$ & $78,70 \%$ & $83,10 \%$ \\
\hline $\begin{array}{c}\text { Desviación } \\
\text { estándar }\end{array}$ & 0,81 & 0,62 & 0,5 & 0,47 & 0,44 \\
\hline
\end{tabular}

Fuente: Elaboración propia

Por una parte, se observa que los resultados obtenidos sobre las preguntas relacionadas con el trabajo que desarrolló cada grupo son bastante buenos, hecho que demuestra una gran implicación de los estudiantes con su propio trabajo. Por otra parte se puede ver que no se logró despertar el interés por los trabajos de los compañeros, ya que en ese caso sólo el $11,6 \%$ de las respuestas es acertada; también se puede ver que los resultados de las preguntas teóricas son bastante bajos.

El bajo resultado en estos dos apartados pueden deberse al poco peso que representa frente a la nota definitiva, da la impresión de que la estrategia que siguen los alumnos es la de llegar al examen final con la asignatura prácticamente aprobada, de esta forma en el período de exámenes dedican más esfuerzo al estudio de las demás asignaturas.

\section{CONCLUSIONES Y LÍNEAS FUTURAS DE INVESTIGACIÓN}

Tal y como se observa en los resultados, mediante la utilización de la metodología descrita el aprendizaje de los contenidos se resuelve de una manera exitosa. El plantearle al alumno que resuelva un problema en una empresa real de una manera semi-autónoma hace que éste se plantee cuestiones que con las clases magistrales no lo haría. Además, debido al seguimiento realizado a través de los hitos por el profesor, los equipos son capaces de corregir sus errores y el conocimiento de la materia se refuerza.

En lo que a otras competencias se refiere, se concluye que se incide sobre el desarrollo de varias de ellas que con los métodos tradicionales de enseñanza no se trabajan: la capacidad de gestionar un proyecto, los problemas de calendario y reparto de tareas que éste conlleva, la capacidad de resolver conflictos, tanto propios del proyecto como los internos del grupo, la capacidad de exponer los resultados en público y la capacidad de defender un trabajo propio.

Este trabajo no está exento de limitaciones: por ejemplo, el análisis por resultados en las notas de los alumnos sería mucho más rico si se hubiera podido realizar una comparación con los resultados antes de haber utilizado esta metodología, y con los resultados globales de los cursos donde se imparten los cursos de Gestión de Operaciones. 
Respecto a investigaciones futuras, los autores se están planteando ampliar este estudio a otras universidades donde los alumnos, en asignaturas del área de Operaciones, también realizan trabajos sobre empresas reales. Este análisis comparativo más amplio ayudaría a obtener resultados más amplios, y las conclusiones podrían ser muy útiles para mejorar los resultados obtenidos en la Tabla 3 de nuestro estudio. Otra línea de trabajo, sería aplicar esta metodología a la asignatura "Trabajo fin de grado", la cual es obligatoria en todos los grados, y donde la realización de un trabajo con una empresa real encaja perfectamente. Un aspecto muy interesante a analizar es unir la metodología de project-based learning con la metodología de investigación Action Research, la cual consiste en realizar investigación que sea relevante tanto desde el punto de vista del practitioner como de los investigadores, tal como se muestra en Coughlan et al., (2001) y Carr (2013).

\section{BIBLIOGRAFÍA}

Barnes, L., Christensen, R., y Hansen, A. [1994A]: Premises and practices of discussion teaching, en L. Barnes, R. Christensen, and A. Hansen (Eds.), Teaching and the case method. Boston: Harvard Business School Press.

BARnes, L., Christensen, R., Y HANSEN, A. [1994B]: Teaching with cases at Harvard Business School, en L. Barnes, R. Christensen, y A. Hansen (Eds.), Teaching and the case method. Boston: Harvard Business School Press.

CARR, D. [2013]: An in-reaching community of practice: Constructing a learning cooperative, using a project based teaching and learning model in a Higher Education. Journal of Innovations in Practice, 8: 61-85.

ChInNOWSKY, P., Brown, H., SZAJNMAN, A. y RealPH, A. [2006]: Developing knowledge landscapes through project-based learning, Journal of Professional Issues in Engineering Education and Practice, vol. 132, núm. 2: 118-125.

CHRISTENSEN, C. R. [1991]: The discussion teacher in action: Questioning, listening, and response, en C. R. Christensen, D. Garvin, y A. Sweet (Eds.), Education for judgment: The artistry of discussion Leadership. Boston: Harvard Business School Press.

CMDSG [2004]: Por una globalización justa: crear oportunidades para todos. Ginebra: OIT. En línea: http://www.llo.org/public/spanish/wcsdg/docs/report.pdf

COUghlan, P., DromgOOLE, T., DufF, D. y HaRIDON, A. [2001]: Continuous Improvement through Collaborative Action Learning, International Journal of Technology Management, vol. 22, núm.4: 285-302.

ERSKINE, J., LeENDERS, M., Y MAUfFETTE-LeENDERS, L. [1998]: Teaching with cases. London/Ont., Canada: Richard Ivey School of Business/The University of Western Ontario.

GIJSELAERS, W. H. [1996]: Connecting problem-based learning with educational theory, en: L. Wilkerson y W. H. Gijselaers (Eds) Bringing problem-based learning to higher education: theory y practice. San Francisco, CA, Jossey-Bass: 13-21.

JACKSON, H., TARHINI, K., MAGGI, B., Y RUMSEY, N. [2012]: Improving Students Understanding of Engineering Concepts Through Project Based Learning. In 2012 Frontiers in Education Conference Proceedings (pp. 1-6). IEEE.

JACKSON, J. [2004]: Case-based teaching in a bilingual context: Perceptions of business faculty, in Hong Kong English for Specific Purposes 23: 213-232

JOHNSON, P.A. [1999]: Project-based, cooperative learning in the engineering classroom, Journal of Professional Issues in Engineering Education and Practice, vol. 125, núm. 1: 8-11.

KeLLY, W. [2007]: Certification and Accreditation in Civil Engineering, Journal of Professional Issues in Engineering Education and Practice, vol. 133, núm, 3: 181187.

MAUfFEtTE-LeENDERS, L., ERSKINE, J., Y LEenders, M. [1997]: Learning with cases, London/Ont., Canada: Richard Ivey School of Business/The University of Western Ontario. 
MULCAHY, D. [2000]: Turning the contradictions of competence: competence-based training and the beyond, Journal of Vocational Education and Training, vol. 52, núm. 2: 259-280.

PADMANADHAN, G. Y KATTI, D. [2002]: Using community-based projects in civil engineering capstone courses, Journal of Professional Issues in Engineering Education and Practice, vol. 125, núm. 1: 12-18.

PARSONS, C., CAYLOR, E. Y SIMMONS, H. [2005]: Cooperative Education Work Assignments The role of Organizational and Individual Factors in Enhancing ABET Competencies and $\mathrm{Cp}$-op Workplace Well-Being, Journal of Engineering Education, vol. 94, núm. 3: 309-316.

PRONKO, M. [2005]: Recomendación 195 de OIT: Cuestiones históricas y actuales de la formación profesional. Montevideo: Cinterford/OIT.

WELTY, W. [1989]: Discussion method teaching: How to make it work, Change (July/August): 41-49. 